\title{
Gallbladder carcinoma - when to use laparoscopic approach?
}

\author{
Bakos $\mathrm{M}^{1}$, Jankovic $\mathrm{T}^{1}$, Durdik $\mathrm{S}^{2}$, Vrtik $\mathrm{L}^{3}$ \\ Department of Surgery, University Hospital Nitra, Slovakia. marianbakos@atlas.sk
}

\begin{abstract}
AIM: To assess how laparoscopy has altered the presentation of patients with gallbladder cancer and determine whether radical resection in patients with gallbladder cancer is beneficial.

METHOD: 47 patients underwent surgery because of suspected gallbladder cancer. Cancer was found incidentally in 29 patients (61.7\%) during routine laparoscopic cholecystectomy using frozen biopsy. Gallbladder cancer had been diagnosed preoperatively in the other 18 patients (38.3\%).

RESULTS: Patients in whom carcinoma was found incidentally at laparoscopic cholecystectomy had a significant increase in survival when compared with those who were admitted electively with a known diagnosis. All patients who presented with a known diagnosis had stage II or higher, and $38.3 \%$ of these were in stage IV. However, $58.6 \%$ of those patients who were found incidentally were in stage I or II. The overall 2-year survival for all patients was $45 \%$; those discovered incidentally at laparoscopic cholecystectomy (Tis-T2) had a 2-year survival of $87 \%$.

CONCLUSION: Laparoscopic cholecystectomy resulted in an earlier discovery of gallbladder cancer in some patients, resulting in increased probability of survival. Adjunctive radical surgical resection, either at the time of cholecystectomy or subsequently, increases survival significantly in early stage disease (Tab.2, Fig. 2, Ref. 24). Text in PDF www.elis.sk KEY WORDS: gallbladder carcinoma, laparoscopic approach.
\end{abstract}

\section{Introduction}

Gallbladder carcinoma, despite of its relative rarity, is the most common cancer of biliary system. There are some discrepancies about this carcinoma and its epidemiology in literary sources. Despite of its rarity with incidence of 1-2/100 000 inhabitants (1), it is an extremely aggressive carcinoma with high mortality, approximately 2500 people every year. General incidence of gallbladder and extrahepatic biliary ducts carcinomas in Europe is 3.2 and 5.4 of 100000 men and women, respectively. Incidence of intrahepatic cholangiocarcinoma increases, and average incidence is $0.9-1.3$ and $0.4-0.7$ per 100000 men and women. This numbers present $10-15 \%$ of all primary tumours of liver. In high risk regions of Europe (south Italy) there is an incidence of 4.9-7.4 and 2.9-4.3 per 100000 men and women, respectively. Worldwide, the highest incidence of this carcinoma is in Thailand, even 96/100 000 (2).

Symptoms of gallbladder carcinoma are not specified, in early stage they can imitate cholecystolithiasis. Gallstones are current in many of patients with gallbladder cancer. Every year more than 750000 patients undergo surgery because of cholecystolithiasis (3). The carcinoma is found in $1 \%$ (or less) of all cholecystectomies (4-6).

${ }^{1}$ Department of Surgery, University Hospital Nitra, Slovakia, ${ }^{2}$ Department of Surgery, Oncology Institute of St. Elizabeth, Bratislava, Slovakia, and ${ }^{3} 1$ st Department of Surgery, Faculty of Medicine UK, Bratislava, Slovakia

Address for correspondence: M. Bakos, MD, PhD, Department of Surgery, University Hospital Nitra, Spitalska 6, SK-950 01 Nitra, Slovakia.
From the beginning of 90's in the last century, laparoscopic cholecystectomy has got its acknowledgment and has expanded all over the world. From this reason also higher incidence of accidentally found gallbladder carcinomas has been detected. Only one potential therapeutic method is its radical resection. Until recently, published results on this topic were not sufficient.

In our study we observed that using laparoscopic approach was helpful in diagnostic process of gallbladder carcinoma, therefore if it changed the management a result of therapy in patients with this carcinoma. Also, we have evaluated if radical laparoscopic resection is appropriate therapy in patients with gallbladder carcinoma.

\section{Materials and methods}

\section{Diagnostic methods}

In the diagnostic process of gallbladder carcinoma it is necessary to consider differential diagnostics and size of tumour. This is the reasons why the imaging methods like MRI, CT or MRCP are especially important. Endoscopic ultrasonography is also exceptionally reliable method in detection of benign pathology of gallbladder $(5,6)$, but it is not used in our clinic.

The most of gallbladder tumours are benign. With USG methods adenomas, cholesterol polyps or adenomyomas are often diagnosed. USG diagnostic of precancerous and malign lesions is more complicated. Polyps with size more than $1 \mathrm{~cm}$ are indicated for prophylactic cholecystectomy. Endoscopic ultrasonography can be an appropriate method in differential diagnostic process and exclusion of tumours in suspicion of polyps or unclear thick- 
Tab. 1. Gallbladder carcinoma classification.

\begin{tabular}{|c|c|c|c|}
\hline \multicolumn{4}{|c|}{ Primary tumour $(\mathrm{T})$} \\
\hline TX & \multicolumn{3}{|c|}{ Primary tumour is not possible to evaluate } \\
\hline T0 & \multicolumn{3}{|c|}{ Without evidence of primary tumour } \\
\hline Tis & \multicolumn{3}{|c|}{ In situ carcinoma } \\
\hline $\mathrm{T} 1$ & \multicolumn{3}{|c|}{ Tumour disseminates into lamina propria or into muscle layer } \\
\hline T1a & \multicolumn{3}{|c|}{ Tumour disseminates into lamina propria } \\
\hline $\mathrm{T} 1 \mathrm{~b}$ & \multicolumn{3}{|c|}{ Tumour disseminates into muscle layer } \\
\hline $\mathrm{T} 2$ & \multicolumn{3}{|c|}{$\begin{array}{l}\text { Tumour disseminates into perimuscular tissue, no penetration trough serosa } \\
\text { or invasion into the liver }\end{array}$} \\
\hline T3 & \multicolumn{3}{|c|}{$\begin{array}{l}\text { Tumour disseminates serosa (visceral peritoneum) and/or directly dissemi- } \\
\text { nates in liver, and/or disseminates into the surrounding structures or organs, } \\
\text { for example stomach, duodenum, gut, pancreas, omentum or extrahepatic ducts }\end{array}$} \\
\hline$\overline{\mathrm{T} 4}$ & \multicolumn{3}{|c|}{$\begin{array}{l}\text { Tumour disseminates into main branch of vena portae or hepatic arteries or } \\
\text { disseminates into two or more extrahepatic structures or organs }\end{array}$} \\
\hline \multicolumn{4}{|c|}{ Regional lymphatic nodes $(\mathrm{N})$} \\
\hline $\mathrm{NX}$ & \multicolumn{3}{|c|}{ Regional lymphatic nodes are not possible to evaluate } \\
\hline N0 & \multicolumn{3}{|c|}{ Without metastases into the regional lymphatic nodes } \\
\hline$\overline{\mathrm{N} 1}$ & \multicolumn{3}{|c|}{$\begin{array}{l}\text { Metastases into the nodes along ductus cysticus, ductus hepaticus com., hepatic } \\
\text { arteries and/or vena portae }\end{array}$} \\
\hline N2 & \multicolumn{3}{|c|}{$\begin{array}{l}\text { Metastases into periaortic nodes, pericaval nodes, into the nodes of arteria } \\
\text { mesenteric sup. and/or into the nodes around celiac arteries }\end{array}$} \\
\hline \multicolumn{4}{|c|}{ Distant metastasis (M) } \\
\hline$\overline{\mathrm{M} 0}$ & \multicolumn{3}{|c|}{ Without distant metastasis } \\
\hline M1 & \multicolumn{3}{|c|}{ Presence of distant metastasis } \\
\hline \multicolumn{4}{|l|}{ Staging } \\
\hline Stage 0 & Tis & N0 & M0 \\
\hline Stage I & $\mathrm{T} 1$ & N0 & M0 \\
\hline Stage II & $\mathrm{T} 2$ & No & M0 \\
\hline Stage IIIA & $\mathrm{T} 3$ & N0 & M0 \\
\hline Stage IIIB & $\mathrm{T} 1-3$ & N1 & M0 \\
\hline Stage IVA & $\mathrm{T} 1-4$ & $\mathrm{~N} 2$ & M0 \\
\hline Stage IVB & $\mathrm{T} 1-4$ & $\mathrm{~N} 0-2$ & M1 \\
\hline
\end{tabular}

stones, but CT images show changes of the wall in locations, which are overlaid with gallstones or calcifications. CT can detect local or irregular thickening of the wall, dilatation of bile ducts, local invasion, metastasis and (8).

\section{Cytology}

Cell cytology from mucosa of gallbladder is quick, simple and high-quality method in carcinoma detection. Ultrasonography navigated aspiration with thin needle is also a safe diagnostic modality. ERCP of biliary system is a specific method and we must use it in unclear and suspicious lesions $(7,8)$.

\section{Markers}

Tumour markers have rising application in diagnostic process of gallbladder carcinoma. Markers such CA242, CA15-3, CA19-9 and CA125 are extremely useful differential method in patients with carcinoma and in patients with cholecystolithiasis. The highest specificity had common evaluation of CA242 a CA125. Electrophoresis of proteins from patient's serum with carcinoma and from patient's with gallstones is a modern method of differential diagnosis.

Diagnosis of gallbladder carcinoma must be established on the basis of radiologic examination (MR, CT) and pathomorphological examination from biopsy, ness of gallbladder wall. CT and MR are used in staging a sizing of tumour or determination of metastasis (5-7) (Tab. 1). Ultrasonography is a very often used method in diagnostic process of patients with icterus or in nonspecific gastrointestinal disorders. It is especially sensitive method in detection of obstruction of bile duct (5). In case of uncertainty or when USG is pointing at resectability of malignancy, CT, MR, MRCP or cholangiography are helpful. For verification of malignancy and for its classification we can use needle biopsy or endoscopic brush (7). The reason of negative prognosis of gallbladder carcinoma is its early dissemination and usually its late diagnostic. Problem is that the patients with this diagnosis have nonspecific clinical symptoms. The first step in early diagnostic is to correct the identification and evaluation of patient's disorders. To increase the sensitivity of USG it is necessary to use right technology and skilled specialist. In cases, when USG detects gallbladder tumour, we can do FDG-PET (fluorodeoxyglucose positron emission tomography) for confirming the diagnosis (benign or malign lesion). If we confirm gallbladder carcinoma, CT is extremely helpful for determination about local dissemination of carcinoma. USG is better method for detection of disparity of gallbladder wall, thickness of gallbladder and gall- needle aspiration or cytology according to classification of World Health Organization (Tab. 1). Pathological diagnosis must be established before start of chemotherapy, radiotherapy or another nonsurgery oncological therapy. But it is not necessary before surgery in patients with resectable tumour.

\section{Surgical technique}

During laparoscopy cholecystectomy was done in patients with suspicion of early stage of carcinoma. By laparoscopic cholecystectomy there are three ports standardly done. After the cholecystectomy, gallbladder was extracted in safety plastic bag through supraumbilical port. Gallbladder was histologically examined and according to the stage, we planned reoperation-resection of liver and lymphadenectomy. If the locoregional lymphadenectomy was done, lymphatic nodes from pericholedocal, hilus, periportal and common hepatic area were removed. We used harmonic scalpel for lymphadenectomy. At first, we removed nodes from area around hepatoduodenal ligamentum, then we proceeded in the area around common hepatic arteria. After careful haemostasis, we used silicon drainage inserted into subhepatic area. Surgery with liver resection and lymphatic nodes, with negative border of 
resected tissue of gallbladder and without metastatic lymphatic nodes was considered as curative.

\section{Postoperative observation and diagnosis of recurrence}

Postoperative patient's observation consisted of physical examination and laboratory tests, including determination of carcinoembryonic antigen and level of CA19-9. Laboratory tests were done 1 time per three months during first two years. Important part of postoperative observation was USG of abdomen or CT.

\section{Results}

Between the years 2015-2019 47 patients with gallbladder carcinoma were retrospectively identified. From 29 patients with incidental carcinoma was carcinoma diagnosed in 25 (53.2\%) during or after laparoscopic cholecystectomy. In 4 patients $(8.5 \%)$ was carcinoma diagnosed during open cholecystectomy. In 17 patients (36.2\%) stage II (or lower) carcinoma was detected, because already cholecystectomy was curative and another surgery wasn't performed. Carcinoma during laparoscopy was found in 3 patients $(6.4 \%)$, therewith after perioperative biopsy and confirmed carcinoma II (or higher) was realised conversion to open surgery. From these patients, in 1case $(2.1 \%)$, the tumour wasn't resectable. Two out of three patients $(4.3 \%)$, in whom conversion was realised, underwent radical resection - resection of liver with lymphadenectomy. One patient (2.1\%), after the diagnosis of carcinoma, refused reoperation and 8 patients $(17 \%)$ underwent reoperation. From these 8 patients, 2 patients $(4.3 \%)$ had nonresectable tumour, so after revision the surgery was ended. Another 6 patients $(12.7 \%)$ underwent reoperation, in 4 cases
Tab. 2. Our patients divided by stage of their gallbladder carcinoma.

\begin{tabular}{lc}
\hline Stage & Number of patients \\
\hline 0 & 2 \\
Ia & 4 \\
Ib & 6 \\
II & 9 \\
III & 8 \\
IV & 18 \\
\hline Total & 47
\end{tabular}

(8.5\%) was resection of liver done with lymphadenectomy and in 2 cases ( $4.3 \%$ ), when it was not possible to do resection, palliative hepatojejunal anastomosis was constructed (Fig. 1).

From 18 patients $(38.3 \%)$, in whom was the carcinoma diagnosed before surgery, $4(8.5 \%)$ had nonresectable tumour, what was founded also before surgery by imaging methods used. No one did refuse surgery. 14 patients $(29.8 \%)$, in whom the tumour seemed resectable, underwent surgery. In 2 cases ( $4.3 \%$ ) nonresectable tumour was found even during the surgery, so the surgery was finished. 12 patients ( $25.5 \%$ ) underwent surgery, in 3 cases $(6.4 \%)$ simple cholecystectomy was realised, because the stage of tumour was II or lower and simple cholecystectomy is for this stage curative. 9 patients $(19.1 \%)$ underwent radical surgery. Because of the stage of tumour, in 2 patients $(4.3 \%)$ resection of liver with lymphadenectomy was realised and in 7 patients (14.9\%) palliative hepatojejunal anastomosis was constructed (Fig. 2).

Our results show that in cases of tumour stage II or lower, the laparoscopic approach is an appropriate method for this diagnosis. Patients with carcinoma stage II are borderline group and therapeutic approach is individual. Important fact is that cholecys-

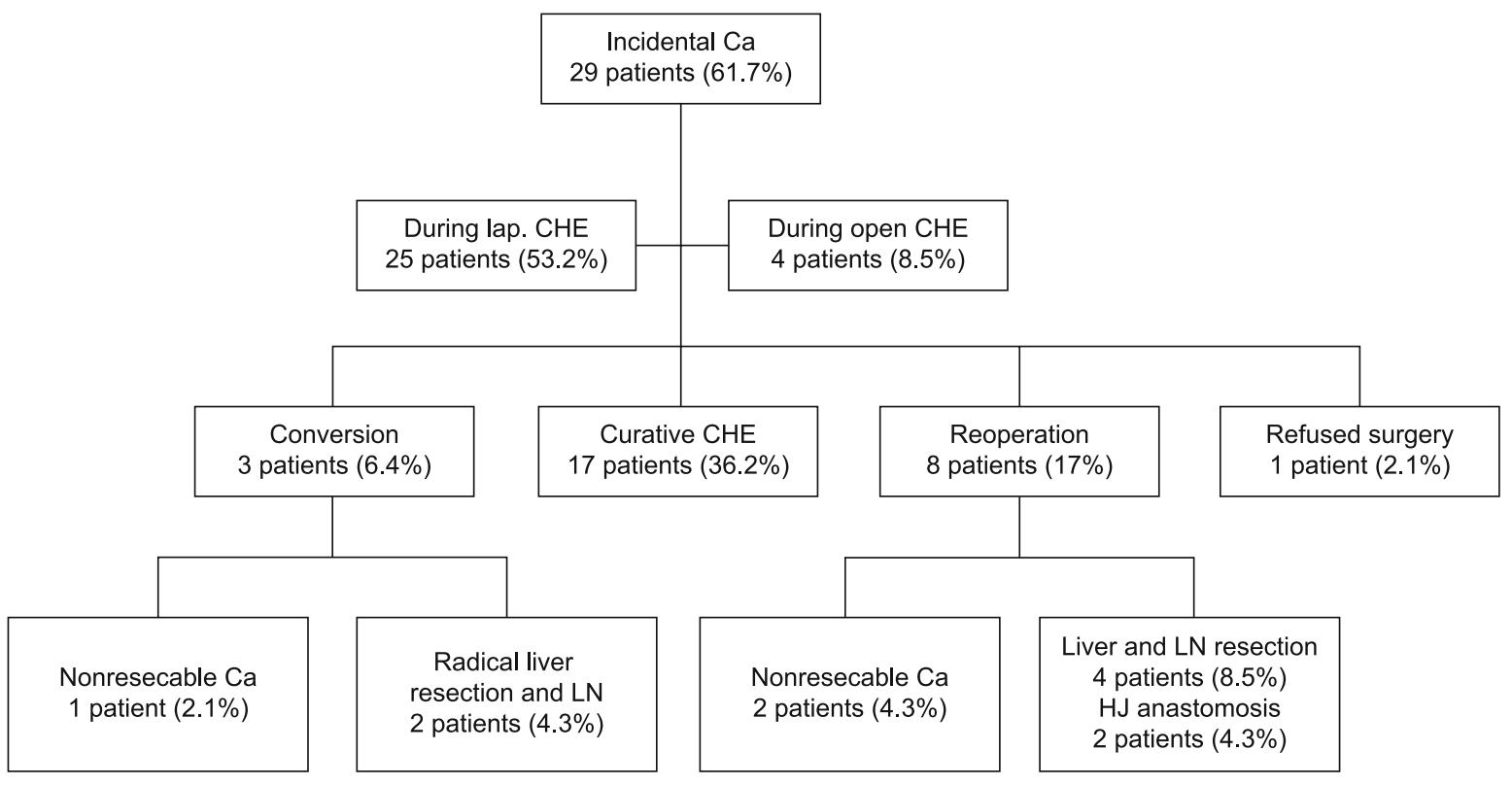

Fig. 1. Diagram for incidental cases of gallbladder carcinoma. 


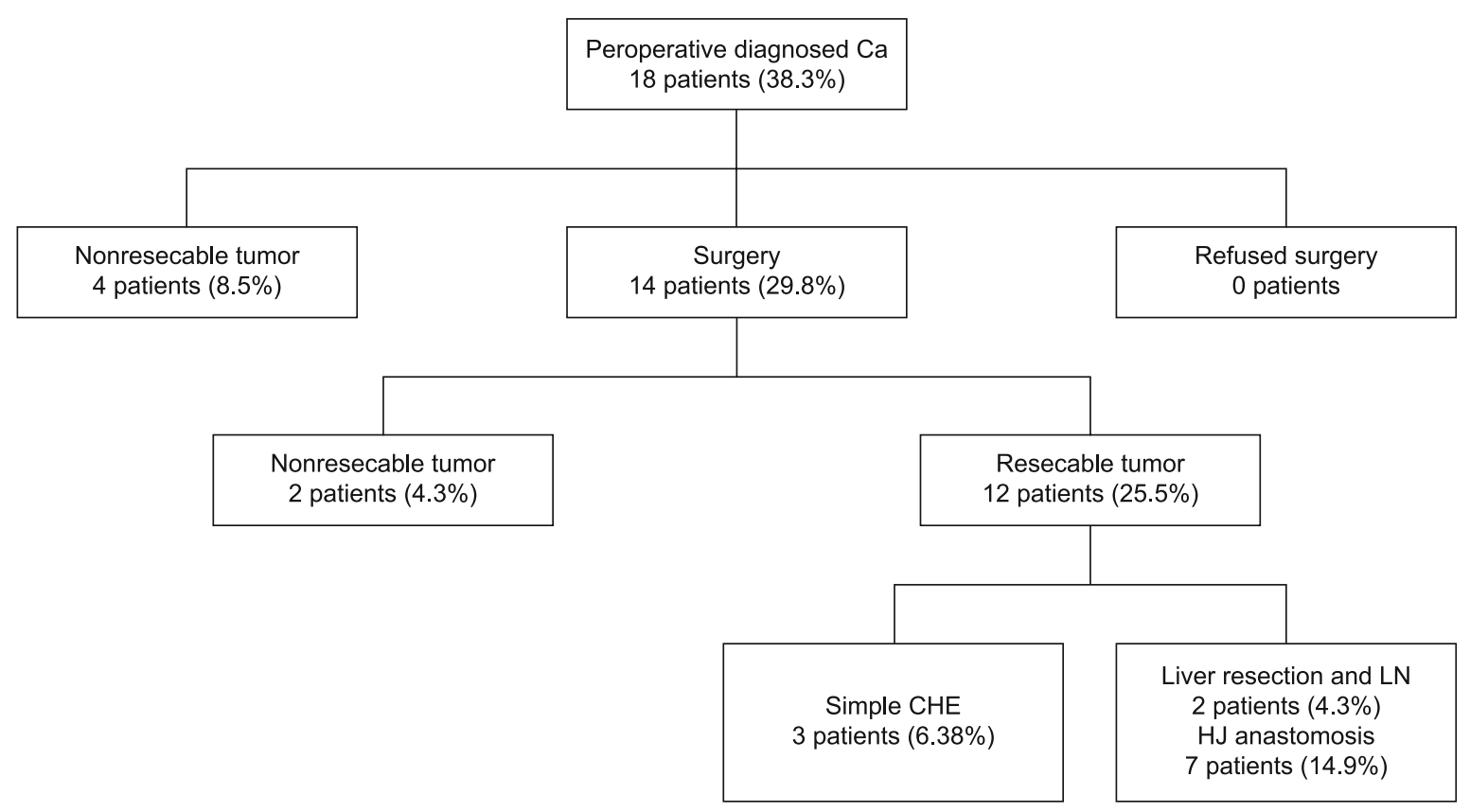

Fig. 2. Diagram for non-incidental cases of gallbladder carcinoma.

tectomy is curative surgery in these patients' groups. In patients with carcinoma stage II or higher, we recommend radical resection - liver and lymphatic nodes resection. In our workplace, we have done this surgery only in classical way. In patients with high stage of carcinoma (IV), after the surgeon rational evaluation of carcinoma, we recommend palliative hepatojejunal anastomosis. This is important when the resection of liver and lymphatic nodes is not curative, because of effort to prolong patient's life. The group of our patients was divided according to carcinoma stage as shown in Table 2.

\section{Discussion}

Laparoscopic approach can be an alternative method in therapy of preoperatively diagnosed early stage gallbladder carcinomas (7). We can come out from concept, that if the carcinoma is detected accidentally, it is probably early stage of carcinoma. This fact improves the chance for patient's treatment. Our observation of patients with stage II has shown, that a better survival is in group where the carcinoma was diagnosed incidentally. In the cases of incidentally detected carcinoma during laparoscopy, it is recommended to do additional reresection (8). Patients with incidentally detected gallbladder carcinoma (most often during laparoscopic cholecystectomy) have better survival against the patients, in whom the carcinoma was diagnosed before surgery. We can use CT without/with PET examination of abdomen and bile ducts and MRI cholangiopancreatography. Planned curative resections (in patients with incidentally detected carcinoma) didn't have negative influence for survival.
Japanese study (Ouchi et al) from more medical centres has published results of 498 patients with gallbladder carcinoma solved with laparoscopic cholecystectomy. They have found, that the perforation of gallbladder during laparoscopy significantly aggravated survival of patients. Also they have found, that additional excisions in patients with $\mathrm{T} 2$ stage (tumour disseminates into perimuscular tissue, no penetration through serosa or invasion into the liver) and with T3 stage (tumour disseminates serosa (visceral peritoneum) and/or directly disseminates into liver, and/ or disseminates into the surrounding structures or organs) improve survival. This surgery technique doesn't carry any significant changes in survival in patients with T1 tumours (tumour disseminates into lamina propria or into muscle layer) and $\mathrm{T} 4$ (tumour disseminates into main branch of vena portae or hepatic arteries or disseminates into two or more extrahepatic structures or organs, invasion into the liver in range of $2 \mathrm{~cm}$ ). 5-years survival of patients with T2 tumours was $70 \%(9)$.

Some studies described only patients, in whom carcinoma was diagnosed incidentally during cholecystectomy. Toyonaga et al. described set of 73 patients between 1982 and 2000, in whom carcinoma was detected even during cholecystectomy. 43 patients had planned secondary radical resection. They defined secondary resection as "liver resection in gallbladder area and dissection of regional lymphatic nodes with or without resection of extrahepatic bile ducts". Overall, 21 patients underwent this type of surgery (18 patients with T2, 3 patients with T3). 5 years survival after secondary resection was $54 \%$ in patients with carcinoma without invasion to another organ, similarly as 5 years survival in patients with carcinoma stage II. From 75 patients, 45 initially underwent 
laparoscopic cholecystectomy, but this fact did not have influence on the results (10).

Bartlett et al. published, that in T2 carcinoma stage there is an incidence of metastasis into the lymphatic nodes $46 \%$ (11). American Cancer Society has published, that in 1994 and 1995, $58.5 \%$ patients underwent simple cholecystectomy with or without dissection of lymphatic nodes, while only $9.8 \%$ underwent cholecystectomy with liver resection with or without dissection of lymphatic nodes (12). These data show that in the past, the most of surgeons didn't use aggressive approach in therapy of this carcinoma. To improve survival of patients with gallbladder carcinoma, surgeons started to use more aggressive techniques.

Dixon et al. has published a study of 99 patients with gallbladder carcinoma. They were treated in two time periods: between 1990-1996 and between 1997-2002.From these patients $25 \%$ underwent laparoscopic cholecystectomy. 39 patients underwent resection, and the result was $\mathrm{R} 0$ resection in 33 patients. 5-years survival was significantly better during second period, because in that period the number of radical resections was higher (35\%) against first period (7\%). Authors concluded that the most important factor for patient long term survival is radical R0 resection (13).

In the situations when the preoperative profiles and perioperative findings marked malignity, the surgical technique and skills are especially important. If the laparoscopic dissection of lymphatic nodes is difficult or there is a high risk of gallbladder perforation, the conversion is recommended. The reason for conversion is better overview and prevention of dissemination of malignant cells. Many published studies confirm this fact, for example new growth of tumour in area of port (14-16). The use of insulating bags decreases dissemination and recurrence $(17,18)$.

Shirai et al. mentioned, that lymphatic drainage includes pericholedocal, pancreaticoduodenal and intraaortocaval lymphatic nodes. Exact range of lymphatic nodes resection by optimal surgery of gallbladder carcinoma has still not been determined (19).

In our workplace, we usually do complete dissection of portal lymphatic nodes and dissection in area of hepatic artery and portal vein. Our study did not show significant difference in patient survival between patients (with liver, gallbladder and lymphatic nodes resection) with or without resection of extrahepatic bile ducts. Laparoscopic approach used in early carcinoma stage (without invasion into liver) has two potential benefits. The first is that laparoscopic approach can be equivalent with open radical cholecystectomy with the same oncology aspect and in future will be used as curative method for early stage carcinoma. The second is that if benign lesions are diagnosed during laparoscopic cholecystectomy, for example andenomyomatosis or xantogranulomatosis cholecystitis, we can avoid of unnecessary open laparotomy.

\section{Conclusions}

Gallbladder carcinoma is one of the most malignant carcinomas of gastrointestinal tract. Its prognosis is the worst and period of survival is the shortest. The only one potential curative method is radical resection. Role of chemotherapy and radiotherapy in the management of treatment is not sufficiently examined. Because of the aggressivity of the tumour and higher stage at the time of diagnosis, the results of therapy are not ideal. Very often gallbladder carcinoma spreads through wall into the surrounding organs, mainly into the liver. From some studies resulted, that carcinoma invasion into the liver is seen in 34-89\% cases. In early carcinoma stages proliferation to lymphatic nodes is frequent $(20,21)$.

Only cholecystectomy is not sufficient in stage pT2, because of positive lymphatic nodes (22). Additional radical resection, which is done during or after cholecystectomy, significantly increase survival of patients (23). Many authors have published, that dissection of lymphatic nodes improves survival of patients in early carcinoma stage $(22,24)$. The surgery of hepatobiliary tract has changed in the last decade. Laparoscopic cholecystectomy, better and sophisticated surgical methods improve management of patients with carcinoma. Laparoscopic approach can be an alternative method for treatment of early stages gallbladder carcinoma. Early diagnosis and aggressive surgical resection are appropriate methods in the treatment of carcinoma. Additional chemo- or radiotherapy will be important factor for positive results in the future.

Our results show that laparoscopic approach is technically possible, and it is acceptable for patients with early carcinoma stages without invasion into the liver.

\section{References}

1. Fong Y, Malhotra S. Gallbladder cancer: recent advances and current guidelines for surgical therapy. Adv Surg 2001; 35: 1-20.

2. Carriaga MT, Henson DE. Liver, gallbladder, extrahepatic bile ducts, and pancreas. Cancer 1995; 75: 171-190.

3. Shaffer EA. Curr Gastroenterol Rep 2005; 7 (2): 132-140.

4. Frauenschuh D, Greim R, Krase E. How to proceed in patients with carcinoma detected after laparoscopic cholecystectomy. Langenbecks Arch Surg 2000; 385: 495-500.

5. Romano F et al. Laparoscopic cholecystectomy and unsuspected gallbladder cancer. Eur J Surg Oncol 2001; 27: 225-228.

6. Wysocki A et al. Laparoscopic cholecystectomy and gallbladder cancer. Surg Endosc 1999; 13: 899 -901

7. Steinle EW et al. Impact of laparoscopic cholecystectomy on indications for surgical treatment of gallstones. Surg Endosc 1997; 11: 933-935.

8. Shirai $\mathbf{Y}$ et al.Inapparent carcinoma of the gallbladder: an appraisal of a radical second operation after simple cholecystectomy. Ann Surg 1992; 215: $326-331$.

9. Ouchi K, Mikuni J, Kakugawa Y. Laparoscopic cholecystectomy for gallbladder carcinoma: results of a Japanese survey of 498 patients. J Hepatobiliary Pancreat Surg 2002; 9: 256-260.

10. Toyonaga $\mathbf{T}$ et al. Completion of radical surgery after cholecystectomy for accidentally undiagnosed gallbladder carcinoma. World J Surg 2002; 26: 867-871. 
$341-346$

11. Bartlett DL et al. Long-term results after resection for gallbladder cancer: implications for staging and management. Ann Surg 1996; 224: 639-646.

12. Donohue JH, Stewart AK, Menck HR. The National Cancer Data Base report on carcinoma of the gallbladder, 1989-1995. Cancer 1998; 83: 2618-2628.

13. Dixon $\mathbf{E}$ et al. An aggressive surgical approach leads to improved survival in patients with gallbladder cancer. Ann Surg 2005; 241: 385-394.

14. Suzuki K, Kimura T, Ogawa H. Is laparoscopic cholecystectomy hazardous for gallbladder cancer? Surgery 1998; 123: 311-314.

15. Lundberg $\mathbf{O}$. Port site metastases after laparoscopic cholecystectomy. Eur J Surg 2000; 585 (Suppl): 27-30.

16. Fong $\mathbf{Y}$ et al. Gallbladder cancer discovered during laparoscopic surgery-potential for iatrogenic dissemination. Arch Surg 1993; 128: $1054-1056$.

17. Drouard F, Delamarre J, Capron JP. Cutaneous seeding of gallbladder cancer after laparoscopic cholecystectomy. N Engl J Med 1991; 325: 1316-1319.
18. Johnson RC et al. Laparoscopic cholecystectomy: incidental carcinoma of the gallbladder with abdominal wall and axillary node metastasis. HPB Surg 1997; 10: $169-171$.

19. Shirai $\mathbf{Y}$ et al. Identification of the regional lymphatic system of the gallbladder by vital staining. Br J Surg 1992; 79: 659-662.

20. Wanebo HJ, Vezeridis MP. Carcinoma of the gallbladder. J Surg Oncol 1993; 3: $134-139$.

21. Ogura Y et al. Radical operations for carcinoma of the gallbladder: present status in Japan. World J Surg 1991; 15: 337-343.

22. Tsukada K et al. Lymph node spread from carcinoma of the gallbladder. Cancer 1997; 80 (4): 661-667.

23. Shih SP et al. Gallbladder cancer: the role of laparoscopy and radical resection. Ann Surg 2007; 245 (6): 893-901.

24. Kondo $S$ et al. Regional and para-aortic lymphadenectomy in radical surgery for advanced gallbladder carcinoma. Br J Surg 2000; 87 (4): $418-422$.

Received October 28, 2020.

Accepted November 5, 2020. 